\title{
ON SOLUTIONS OF CONVOLUTION EQUATIONS IN SPACES OF ULTRADIFFERENTIABLE FUNCTIONS
}

\author{
D. A. POLYAKOVA
}

\begin{abstract}
A representation for a particular and the general solution is established for convolution equations in nonquasianalytic Beurling spaces ultradifferentiable functions of mean type on a finite interval. As a particular case, differential equations of infinite order with constant coefficients are studied.
\end{abstract}

\section{§1. INTRODUCTION}

Let $\omega$ be a weight function, and let $\varphi_{\omega}^{*}$ be the Young conjugate to the function $\varphi_{\omega}(x)=$ $\omega\left(e^{x}\right)$. Finally, let $I=(-a, a)$ be a finite interval in $\mathbb{R}$.

The paper is devoted to a description of solutions of the convolution equation

$$
T_{\mu} f=g
$$

in the nonquasianalytic Beurling space

$$
\mathcal{E}_{(\omega)}^{1}(I)=\left\{f \in C^{\infty}(I): \forall q \in(0,1), \forall l \in(0, a):|f|_{\omega, q, l}=\sup _{j \in \mathbb{N}_{0}} \sup _{|x| \leq l} \frac{\left|f^{(j)}(x)\right|}{e^{q \varphi_{\omega}^{*}(j / q)}}<\infty\right\}
$$

of ultradifferentiable functions (UDF) of mean type determined by the weight $\omega$ on $I$. Here $T_{\mu}$ is the surjective convolution operator that acts linearly and boundedly on $\mathcal{E}_{(\omega)}^{1}(I)$ by the rule

$$
\left(T_{\mu} f\right)(x)=\left\langle\psi_{\mu}, f(x+\cdot)\right\rangle, \quad f \in \mathcal{E}_{(\omega)}^{1}(I), x \in I ;
$$

$\psi_{\mu}$ is a continuous linear functional in $\left(\mathcal{E}_{(\omega)}^{1}(I)\right)^{\prime}$ called usually the symbol of $T_{\mu}$. For simplicity, by the symbol of $T_{\mu}$ we shall mean the Fourier-Laplace transform $\mu$ of $\psi_{\mu}$ rather than this functional itself. So, the symbol is an entire function that is a multiplier for the weighted space of entire functions

$$
H_{(\omega), I}^{1}=\left\{f \in H(\mathbb{C}) \mid \exists q \in(0,1), \exists l \in(0, a):\|f\|_{\omega, q, l}=\sup _{z \in \mathbb{C}} \frac{|f(z)|}{e^{q \omega(z)+l|\operatorname{Im} z|}}<\infty\right\},
$$

which is isomorphic to $\left(\mathcal{E}_{(\omega)}^{1}(I)\right)^{\prime}$.

It is known (see [1, Theorem 2]) that, as a partial case, equations (1.1) include differential equations of infinite order with constant coefficients

$$
\sum_{k=0}^{\infty} a_{k} f^{(k)}=g, \quad f, g \in \mathcal{E}_{(\omega)}^{1}(I) .
$$

Earlier, in [1, all symbols $\mu$ of the surjective operators $T_{\mu}$ were described, i.e., of all convolution equations (1.1) solvable in $\mathcal{E}_{(\omega)}^{1}(I)$ for every right-hand side $g \in \mathcal{E}_{(\omega)}^{1}(I)$.

2010 Mathematics Subject Classification. Primary 44A35; Secondary 46E10.

Key words and phrases. Ultradifferentiable functions, convolution equations, differential equation of infinite order, series of exponentials.

Supported by RFBR (grant no. 14-01-31083). 
Next, in [2] an exponential polynomial basis was constructed in $\operatorname{ker} T_{\mu}$, i.e., in the space of solutions of the homogeneous differential equation $T_{\mu} f=0$.

In the present paper, we establish the form of a particular solution of (1.1). Together with the results of [2, this enables us to write out the general solution of equations (1.1) and (1.2) in $\mathcal{E}_{(\omega)}^{1}(I)$.

Presently, convolution equations have been studied fairly well also in Beurling spaces of UDF of maximal type (see, e.g., [3, 4, 5] ). However, the form of a particular and the general solution in such spaces has not been established.

A particular solution for equation (1.1) will be sought in the form $f=\sum_{j=1}^{\infty} f_{j} e^{-i \nu_{j} x}$, where the exponents $\nu_{j}, j \in \mathbb{N}$, are chosen in such a way that the system $\left\{e^{-i \nu_{j} x}: j \in \mathbb{N}\right\}$ be an absolutely representative system in $\mathcal{E}_{(\omega)}^{1}(I)$ (an ARS, for short) and the $\left|\mu\left(\nu_{j}\right)\right|$, $j \in \mathbb{N}$, obey appropriate lower estimates. Finally, we shall prove that if $\sum_{j=1}^{\infty} g_{j} e^{-i \nu_{j} x}$ is an expansion of the right-hand side $g$ in (1.1) in an absolutely convergent series, then the function

$$
f=\sum_{j=1}^{\infty} \frac{g_{j}}{\mu\left(\nu_{j}\right)} e^{-i \nu_{j} x}
$$

is a solution for (1.1) in $\mathcal{E}_{(\omega)}^{1}(I)$. This is the principal result of the paper.

In order to construct the system $\left\{\nu_{j}: j \in \mathbb{N}\right\}$, we use the theory of weakly sufficient sets, the theory of ARS, and the relationship between them. The idea of finding a particular solution in a form similar to that indicated above was proposed by Leont'ev in [6]. Later, the method was applied by Korobernik in [7]. This method was also used in [8] for the space $\mathcal{E}_{(\omega)}^{1}(\mathbb{R})$ of Beuring UDF of mean type on the real line.

It should be noted that, in general, the system $\left\{\nu_{j}: j \in \mathbb{N}\right\}$ is found in a nonconstructive way. In this connection, we shall also study the solutions of convolution equations in the spaces $\mathcal{E}_{(\omega)}^{1}(I)$ determined by the weights $\omega(t)=t^{\rho(t)}$, where $\rho(t) \rightarrow \rho \in(0,1)$ is a proximate order. It is known (see [1]) that all convolution equations (1.1) in these spaces are differential equations (1.2) with constant coefficients. If $\rho(t) \equiv \rho \in(0,1)$, then the corresponding spaces $\mathcal{E}_{(\omega)}^{1}(I)$ are projective analogs of the well-known Gevrey classes.

For the class of weights indicated above, the system $\left\{\nu_{j}: j \in \mathbb{N}\right\}$ is constructed explicitly in terms of the symbol $\mu$ of equation (1.2); moreover, the $\nu_{j}$ can be chosen nonnegative. In this construction, we agree to determine $\mu$ by its simple zeros $\left(\lambda_{s}\right)_{s}$ that form an $R$-set (this ensures the surjectivity of $T_{\mu}$ ). Finally, we shall prove that the function (1.3) is a particular solution of equation (1.2), and its general solution has the form

$$
f=\sum_{s=1}^{\infty} a_{s} e^{-i \lambda_{s} x}+\sum_{j=1}^{\infty} \frac{g_{j}}{\mu\left(\nu_{j}\right)} e^{-i \nu_{j} x}, \quad a_{s} \in \mathbb{C}, \quad s=1,2, \ldots
$$

The paper is organized as follows. In $\S 2$, we introduce weight functions, the corresponding Beuring spaces of UDF of mean type, and the dual spaces $H_{(\omega), I}^{1} H_{(\omega), I}^{1}$ and the corresponding ARS of exponentials in $\mathcal{E}_{(\omega)}^{1}(I)$. In $\S 4$, we prove the main result, i.e., the theorem about a partial solution for equation (1.1) in $\mathcal{E}_{(\omega)}^{1}(I)$. $\S \S 5,6$ are devoted to solutions of convolution equations in generalized projective Gevrey classes.

\section{§2. WEIGHT FUnCTIONS, SPACES OF UDF, AND CONVOLUTION OPERATORS}

Let $\omega$ be a nonquasianalytic weight function, i.e., a monotone nondecreasing continuous function $\omega:[0, \infty) \rightarrow[0, \infty)$ satisfying the following conditions:

$$
\text { ( } \alpha) \quad \forall p>1 \exists C>0: \omega(x+y) \leq p(\omega(x)+\omega(y))+C, \quad \forall x, y \geq 0 ;
$$


(ß) $\quad \int_{1}^{\infty} \frac{\omega(t)}{t^{2}} d t<\infty$

$(\gamma) \quad \ln t=o(\omega(t)), \quad t \rightarrow \infty$

$(\delta)$ the function $\varphi_{\omega}(x):=\omega\left(e^{x}\right)$ is convex on $[0, \infty)$.

There is no loss of generality in assuming that $\omega(1)=0$. We put $\omega(z):=\omega(|z|), z \in \mathbb{C}$.

We describe the properties of weight functions required in what follows. It is known that $\omega(t)=o(t)$ as $t \rightarrow \infty$ and

$$
\lim _{r \downarrow 1} \limsup _{t \rightarrow \infty} \frac{\omega(r t)}{\omega(t)}=1 .
$$

Next, for any nonquasianalytic weight $\omega$, an important role is played by its harmonic extension to the upper and lower half-planes:

$$
P_{\omega}(x+i y):= \begin{cases}\frac{|y|}{\pi} \int_{-\infty}^{\infty} \frac{\omega(t)}{(t-x)^{2}+y^{2}} d t & \text { if } y \neq 0 \\ \omega(x) & \text { if } y=0 .\end{cases}
$$

The function $P_{\omega}$ is continuous and subharmonic on the entire plane, moreover, $P_{\omega}(z) \geq$ $\omega(z)$ for all $z \in \mathbb{C}$. We shall need the upper estimates for $P_{\omega}(z)$ established in the next lemma.

Lemma 1. Let $P_{\omega}$ be the harmonic extention of a nonquasianalytic weight $\omega$. Then for every $\varepsilon>0$ there exists $C>0$ such that

$$
P_{\omega}(x+i y) \leq(1+\varepsilon) \omega(x)+\varepsilon|y|+C, \quad x+i y \in \mathbb{C} .
$$

Proof. Fix $\varepsilon \in(0, \pi / 2-1)$. By $(\alpha)$, there exists $C_{1}>0$ with

$$
\omega(x+y) \leq(1+\varepsilon)(\omega(x)+\omega(y))+C_{1}, \quad x, y \geq 0 .
$$

Clearly, it suffices to prove (2.2) for $y>0$. For such $y$, we have

$$
P_{\omega}(x+i y)=\frac{1}{\pi} \int_{-\infty}^{\infty} \frac{\omega(x+y t)}{t^{2}+1} d t \leq(1+\varepsilon) \omega(x)+(1+\varepsilon) \frac{2}{\pi} \int_{0}^{\infty} \frac{\omega(y t)}{t^{2}+1} d t+C_{1} .
$$

Next,

$$
\frac{2}{\pi} \int_{0}^{\infty} \frac{\omega(y t)}{t^{2}+1} d t=\frac{2}{\pi} \int_{0}^{1} \frac{\omega(y t)}{t^{2}+1} d t+\frac{2}{\pi} \int_{1}^{\infty} \frac{\omega(y t)}{t^{2}+1} d t \leq \frac{\omega(y)}{2}+\frac{2 y}{\pi} \int_{y}^{\infty} \frac{\omega(s)}{s^{2}} d s .
$$

Since $\omega(y)=o(y)$ as $y \rightarrow \infty$ and $\int_{y}^{\infty} \frac{\omega(s)}{s^{2}} d s \rightarrow 0$ as $y \rightarrow \infty$ by $(\beta)$, we see that there exists $C_{2}>0$ such that

$$
\frac{\omega(y)}{2}+\frac{2 y}{\pi} \int_{y}^{\infty} \frac{\omega(s)}{s^{2}} d s \leq \frac{\varepsilon}{1+\varepsilon} y+C_{2}
$$

for all $y>0$. Returning to (2.3), finally we obtain

$$
P_{\omega}(x+i y) \leq(1+\varepsilon) \omega(x)+\varepsilon y+C_{1}+(1+\varepsilon) C_{2}, \quad x \in \mathbb{R}, \quad y>0,
$$

proving the lemma.

We pass to the construction of spaces of UDF. Let $\varphi_{\omega}^{*}(y):=\sup \left\{x y-\varphi_{\omega}(x): x \geq 0\right\}$, $y \geq 0$, be the Young conjugate function to $\varphi_{\omega}$. The Beurling space of UDF of mean type on a finite interval $I=(-a, a)$ in $\mathbb{R}$ is defined to be the following weighted space of infinitely differentiable functions on $I$ :

$$
\mathcal{E}_{(\omega)}^{1}(I)=\left\{f \in C^{\infty}(I): \forall q \in(0,1), \forall l \in(0, a):|f|_{\omega, q, l}=\sup _{j \in \mathbb{N}_{0}} \sup _{|x| \leq l} \frac{\left|f^{(j)}(x)\right|}{e^{q \varphi_{\omega}^{*}(j / q)}}<\infty\right\} .
$$


This space possesses a natural topology determined by the collection of seminorms $\left\{|\cdot|_{\omega, q, l}: q \in(0,1), l \in(0, a)\right\}$, and it is an $(F S)$-space with this topology.

As is well known (see 9, Theorem 1]), the Fourier-Laplace transformation of the functionals

$$
F: \varphi \in\left(\mathcal{E}_{(\omega)}^{1}(I)\right)^{\prime} \mapsto \widehat{\varphi}(z):=\varphi_{x}\left(e^{-i x z}\right), \quad z \in \mathbb{C},
$$

establishes a topological isomorphism between the strong dual $\left(\mathcal{E}_{(\omega)}^{1}(I)\right)_{\beta}^{\prime}$ to $\mathcal{E}_{(\omega)}^{1}(I)$ and the following space of entire functions:

$$
H_{(\omega), I}^{1}=\left\{f \in H(\mathbb{C}) \mid \exists q \in(0,1), \exists l \in(0, a):\|f\|_{\omega, q, l}=\sup _{z \in \mathbb{C}} \frac{|f(z)|}{e^{q \omega(z)+l|\operatorname{Im} z|}}<\infty\right\} .
$$

The space $H_{(\omega), I}^{1}$ is endowed with the natural topology of the inductive limit

$$
\text { ind } \operatorname{ind}_{q \in(0,1)} H_{\omega, q, l}
$$

of the Banach spaces $H_{\omega, q, l}=\left\{f \in H(\mathbb{C}):\|f\|_{\omega, q, l}<\infty\right\}$ and becomes a (DFS)-space.

It is easily seen that $H_{(\omega), I}^{1}$ is not closed under multiplication of functions. In accordance with [1, Proposition 1], the set of all multipliers of $H_{(\omega), I}^{1}$, i.e., of all entire functions $\mu$ with $\mu H_{(\omega), I}^{1} \subset H_{(\omega), I}^{1}$ coincides with

$$
M_{(\omega)}^{1}=\left\{\mu \in H(\mathbb{C}): \forall \varepsilon>0 \quad \sup _{z \in \mathbb{C}} \frac{|\mu(z)|}{e^{\varepsilon \omega(z)+\varepsilon|\operatorname{Im} z|}}<\infty\right\} .
$$

Moreover, every multiplier $\mu \in M_{(\omega)}^{1}$ is continuous, i.e., the multiplication operator $\Lambda_{\mu}: f \mapsto \mu f$ acts continuously on $H_{(\omega), I}^{1}$.

Lemma 1 in [1] allows us to replace $\omega(z)$ by $\omega(\operatorname{Re} z)$ in the definitions of the spaces $H_{(\omega), I}^{1}$ and $M_{(\omega)}^{1}$.

A convolution operator in $\mathcal{E}_{(\omega)}^{1}(I)$ is defined as the adjoint to the multiplication operator $\Lambda_{\mu}$, which acts linearly and continuously in $H_{(\omega), I}^{1} \simeq\left(\mathcal{E}_{(\omega)}^{1}(I)\right)_{\beta}^{\prime}$. Specifically, for a nontrivial multiplier $\mu \in M_{(\omega)}^{1}$, we find the continuous linear functional $\psi_{\mu}:=F^{-1}(\mu)$ on $\mathcal{E}_{(\omega)}^{1}(I)$ and for $f \in \mathcal{E}_{(\omega)}^{1}(I)$ put

$$
\left(T_{\mu} f\right)(x):=\left\langle\psi_{\mu}, f(x+\cdot)\right\rangle, \quad x \in I .
$$

This operator $T_{\mu}$ is called the convolution operator with symbol $\mu$. We have $T_{\mu}=$ $\left(F^{-1} \circ \Lambda_{\mu} \circ F\right)^{\prime}$, so $T_{\mu}$ acts linearly and continuously in $\mathcal{E}_{(\omega)}^{1}(I)$.

Note that $T_{\mu}$ acts in the following way on the elements of the system $\left\{e^{-i z x}: z \in \mathbb{C}\right\}$ (which is complete in $\left.\mathcal{E}_{(\omega)}^{1}(I)\right)$ :

$$
T_{\mu}\left(e^{-i z x}\right)=\mu(z) e^{-i z x}, \quad x \in I, \quad z \in \mathbb{C} .
$$

It is known (see [1, Theorem 2]) that if an entire function $\mu(z)=\sum_{k=0}^{\infty} a_{k}(-i)^{k} z^{k}$ is a strong multiplier of $H_{(\omega), I}^{1}$, i.e., satisfies the condition

$$
\sup _{z \in \mathbb{C}} \frac{|\mu(z)|}{e^{\varepsilon \omega(z)}}<\infty \text { for every } \varepsilon>0,
$$

then $T_{\mu} f=\sum_{k=0}^{\infty} a_{k} f^{(k)}$ for all $f \in \mathcal{E}_{(\omega)}^{1}(I)$, i.e., the convolution operator is a differential operator of infinite order with constant coefficients.

In [1, Theorem 3] it was shown that in the case of a strict nonquasianalytic weight (i.e., a weight satisfying $\lim \sup _{t \rightarrow \infty} \frac{\omega(K t)}{\omega(t)}<K$ with some $K>1$ ) all multipliers in $M_{(\omega)}^{1}$ are strong, and all convolution operators are differential operators with constant coefficients. 
In 1, Theorem 1] several equivalent conditions were found that are necessary and sufficient for $T_{\mu}$ to be a surjection of $\mathcal{E}_{(\omega)}^{1}(I)$ onto $\mathcal{E}_{(\omega)}^{1}(I)$. We reproduce one of them, which will be required in what follows:

(SC) for every $\varepsilon>0$ and every $\delta>0$, there exists $r_{0}>0$ such that for every $z=x+i y \in \mathbb{C}$ with $|x| \geq r_{0}$ and $|y| \leq \delta|x|$ there is a circle $C_{z}$ of radius $R_{z} \leq \delta \omega(x)+\delta|y|$ enclosing $z$ and such that all points $\zeta$ on this circle satisfy

$$
|\mu(\zeta)| \geq e^{-\varepsilon \omega(\operatorname{Re} \zeta)-\varepsilon|\operatorname{Im} \zeta|} .
$$

Condition (SC) is equivalent to the statement that $\mu$ is a divisor for $H_{(\omega), I}^{1}$. This means that if $f \in H_{(\omega), I}^{1}$ and $\frac{f}{\mu} \in H(\mathbb{C})$, then $\frac{f}{\mu} \in H_{(\omega), I}^{1}$ (see the paper quoted above).

So, let $\mu$ be a divisor for $H_{(\omega), I}^{1}$. Then the convolution equation (1.1) has a solution in $\mathcal{E}_{(\omega)}^{1}(I)$ for every right-hand side $g \in \mathcal{E}_{(\omega)}^{1}(I)$.

For every $g \in \mathcal{E}_{(\omega)}^{1}(I)$, the general solution of equation (1.1) is the sum of some particular solution and the general solution of the corresponding homogeneous equation

$$
T_{\mu} f=0 .
$$

The general solution of (2.4) was constructed in [2]. Namely, it it easily seen that if $\lambda_{s}$ is a zero of the symbol $\mu$ of the equation in question and $k_{s}$ is the multiplicity of this zero, then the exponential monomials

$$
x^{l} e^{-i \lambda_{s} x}, \quad l=0,1, \ldots, k_{s}-1,
$$

are solutions of the homogeneous equation (2.4). In [2], a special open covering $\left(U_{j}\right)_{j}$ of the zero set $\left(\lambda_{s}\right)_{s}$ for $\mu$ was constructed. Then it was proved that the space of solutions for the homogeneous equation (2.4) has an absolute basis formed by linear combinations of solutions (2.5): the linear combinations are composed of solutions that correspond to the zeros $\lambda_{s}$ belonging to one and the same $U_{j}$.

Thus, the construction of the general solution for (1.1) reduces to the construction of its particular solution.

\section{§3. WEAKLY SUFFICIENT SETS FOR $H_{(\omega), I}^{1}$}

The present section is devoted to the construction of a weakly sufficient set for $H_{(\omega), I}^{1}$ on which $|\mu|$ admits an appropriate lower estimate. Here $\mu$ is a divisor $H_{(\omega), I}^{1}$, i.e., the symbol of a resolvable convolution equation (1.1).

Recall that, by [10, a set $S \subset \mathbb{C}$ is said to be weakly sufficient for $H_{(\omega), I}^{1}$ if the topology $\operatorname{ind}_{q \in(0,1)} \operatorname{ind}_{l \in(0, a)} H_{\omega, q, l ; S}$ coincides with the initial topology $\operatorname{ind}_{q \in(0,1)} \operatorname{ind}_{l \in(0, a)} H_{\omega, q, l}$ of $H_{(\omega), I}^{1} ;$ here

$$
H_{\omega, q, l ; S}=\left\{f \in H_{(\omega), I}^{1}:\|f\|_{\omega, q, l ; S}=\sup _{z \in S} \frac{|f(z)|}{\left.e^{q \omega(\operatorname{Re} z)+l|\operatorname{Im} z|}<\infty\right\} .}\right.
$$

The required weakly sufficient set for $H_{(\omega), I}^{1}$ will be constructed in two stages.

Lemma 2. The set $S_{0}:=\mathbb{R} \cup(i \mathbb{R})$ (i.e., the union of the real and imaginary axes) is weakly sufficient for $H_{(\omega), I}^{1}$.

Proof. The condition that $S_{0}$ is weakly sufficient for $H_{(\omega), I}^{1}$ is equivalent to the requirement that for every $q \in(0,1)$ and $l \in(0, a)$ there exist $\widetilde{q} \in(0,1), \widetilde{l} \in(0, a)$, and $C>0$ such that

$$
\|f\|_{\omega, \widetilde{q}, \tilde{l}} \leq C \cdot\|f\|_{\omega, q, l ; S_{0}} \text { for all } f \in H_{\omega, q, l ; S_{0}}
$$


Fix $q \in(0,1)$ and $l \in(0, a)$. For $f \in H_{\omega, q, l ; S_{0}}$ we have

$$
\begin{aligned}
|f(x)| & \leq\|f\|_{\omega, q, l ; S_{0}} \cdot e^{q \omega(x)}, \quad x \in \mathbb{R} ; \\
|f(i y)| & \leq\|f\|_{\omega, q, l ; S_{0}} \cdot e^{l|y|}, \quad y \in \mathbb{R} .
\end{aligned}
$$

By the Phragmen-Lindelöf principle (see [11, Theorem 6.5.4]), for all $z=x+i y$ with $y \neq 0$ we have

$$
\ln |f(x+i y)| \leq \frac{|y|}{\pi} \int_{-\infty}^{\infty} \frac{\ln |f(t)|}{(x-t)^{2}+y^{2}} d t+D|y|
$$

where $D=\lim \sup _{r \rightarrow \infty} \frac{2}{\pi r} \int_{0}^{\pi} \ln \left|f\left(r e^{i \theta}\right)\right| \sin \theta d \theta$.

By (3.1), we obtain

$$
\frac{|y|}{\pi} \int_{-\infty}^{\infty} \frac{\ln |f(t)|}{(x-t)^{2}+y^{2}} d t \leq \ln \|f\|_{\omega, q, l ; S_{0}}+q P_{\omega}(x+i y) .
$$

To estimate $D$, we consider the indicator $h_{f}(\theta)$ (at the order 1 ) of the entire function $f$. Since $\frac{\omega(r)}{r} \rightarrow 0$ as $r \rightarrow \infty$, from (3.1) and (3.2) it follows that $h_{f}(0)=h_{f}(\pi)=0$, and $h_{f}\left(\frac{\pi}{2}\right) \leq l$. Recalling that the indicator is trigonometrically convex, we conclude that $h_{f}(\theta) \leq l \sin \theta$ for all $\theta \in[0, \pi]$. Consequently, for every $\varepsilon>0$ there exists $r_{\varepsilon}>0$ with

$$
\ln \left|f\left(r e^{i \theta}\right)\right| \leq(l \sin \theta+\varepsilon) r, \quad r \geq r_{\varepsilon}, \quad \theta \in[0, \pi] .
$$

Therefore, for $r \geq r_{\varepsilon}$ we have

$$
\frac{2}{\pi r} \int_{0}^{\pi} \ln \left|f\left(r e^{i \theta}\right)\right| \sin \theta d \theta \leq \frac{2}{\pi} \int_{0}^{\pi}(l \sin \theta+\varepsilon) \sin \theta d \theta=l+\frac{4 \varepsilon}{\pi} .
$$

Thus, $D \leq l$.

Returning to (3.3) and taking (3.4) into account, we see that

$$
\ln |f(x+i y)| \leq \ln \|f\|_{\omega, q, l ; S_{0}}+q P_{\omega}(x+i y)+l|y|
$$

for all $x+i y \in \mathbb{C}$ with $y \neq 0$. Take $\varepsilon>0$ in such a way that $(1+\varepsilon) q<1$ and $l+q \varepsilon<a$. Using Lemma 1, we fix $C>0$ such that (2.2) is fulfilled. As a result, we arrive at

$$
\ln |f(x+i y)| \leq(1+\varepsilon) q \omega(x)+(l+q \varepsilon)|y|+C+\ln \|f\|_{\omega, q, l ; S_{0}}, \quad y \neq 0 .
$$

Putting $\widetilde{q}:=(1+\varepsilon) q$ and $\widetilde{l}:=l+q \varepsilon$, finally we obtain

$$
|f(x+i y)| \leq e^{C} \cdot\|f\|_{\omega, q, l ; S_{0}} \cdot e^{\widetilde{q} \omega(x)+\widetilde{l}|y|}, \quad y \neq 0 .
$$

By continuity, this estimate is true also for $y=0$.

Thus,

and the lemma follows.

$$
\|f\|_{\omega, \widetilde{q}, \tilde{l}} \leq e^{C} \cdot\|f\|_{\omega, q, l ; S_{0}}
$$

Now, taking two sequences $\varepsilon_{k} \downarrow 0$ and $\delta_{k} \downarrow 0$, we use condition (SC) to find the corresponing $r_{k} \uparrow \infty$. We assume that all $r_{k}$ are so large that $\omega(t) \leq t$ for $t \geq r_{1}$ and $2 \delta_{k} r_{k} \geq r_{1}$.

By (SC), every point $z=\operatorname{Re} z$ of the real axis can be put inside a circle $C_{z}^{\mathrm{Re}}$ of radius $R_{z} \leq \delta_{k} \omega(\operatorname{Re} z)$ all points of which satisfy

$$
|\mu(\zeta)| \geq e^{-\varepsilon_{k} \omega(\operatorname{Re} \zeta)-\varepsilon_{k}|\operatorname{Im} \zeta|} .
$$

Moreover, for $\zeta \in C_{z}^{\mathrm{Re}}$ we also have

$$
\begin{aligned}
& |\operatorname{Re} \zeta| \leq|\operatorname{Re} z|+2 R_{z} \leq|\operatorname{Re} z|+2 \delta_{k} \omega(\operatorname{Re} z) \leq\left(1+2 \delta_{k}\right)|\operatorname{Re} z| \\
& |\operatorname{Im} \zeta| \leq 2 R_{z} \leq 2 \delta_{k} \omega(\operatorname{Re} z) .
\end{aligned}
$$


Next, the entire function $\mu$ has zero type at the order 1 , so it is a function of completely regular growth. Thus, outside a certain exceptional set $\bigcup_{j} E_{j}$ of zero linear density, $E_{j}$ is the disk $\left\{z:\left|z-\xi_{j}\right|<\rho_{j}\right\}$, we have $\frac{\ln |\mu(z)|}{|z|} \rightarrow 0$ as $|z| \rightarrow \infty$. It is well known that the disks $E_{j}$ may be taken mutually disjoint. So, we may assume that the $r_{k}, k \in \mathbb{N}$, are chosen so as to obey the conditions

$$
|\mu(z)| \geq e^{-\varepsilon_{k}|z|}, \quad|z| \geq r_{k}, \quad z \notin \bigcup_{j} E_{j} .
$$

In particular, for all points $z=i \operatorname{Im} z$ on the imaginary axis with $r_{k} \leq|\operatorname{Im} z|<r_{k+1}$, $k \in \mathbb{N}, z \notin \bigcup_{j} E_{j}$, we have

$$
|\mu(i \operatorname{Im} z)| \geq e^{-\varepsilon_{k}|\operatorname{Im} z|} .
$$

Now let $z \in \bigcup_{j} E_{j}$. We denote by $j(z)$ the index of the disk containing $z$. Since the set $E_{j}$ of the disks has zero linear density, we have

$$
\lim _{\substack{z \in \bigcup_{j}^{\infty} \\ z \in E_{j}}} \frac{\rho_{j(z)}}{|z|}=0
$$

Consequently, we may assume that

$$
\rho_{j(z)} \leq \delta_{k}|z|, \quad|z| \geq r_{k}, \quad z \in E_{j(z)}
$$

Lemma 3. The set

$$
S=\left[-r_{1}, r_{1}\right] \cup\left[-i r_{1}, i r_{1}\right] \cup\left(\bigcup_{k \in \mathbb{N}} \bigcup_{\substack{z=\operatorname{Re} z \\ r_{k} \leq|\operatorname{Re} z|<r_{k+1}}} C_{z}^{\mathrm{Re}}\right) \cup\left(i \mathbb{R} \backslash \bigcup_{j} E_{j}\right)
$$

is weakly sufficient for $H_{(\omega), I}^{1}$.

Proof. By Lemma 2, it suffices to prove that for every $q \in(0,1)$ and $l \in(0, a)$ there exist $\widetilde{q} \in(0,1), \widetilde{l} \in(0, a)$, and $C>0$ such that

$$
\|f\|_{\omega, \widetilde{q}, \widetilde{l} ; S_{0}} \leq C \cdot\|f\|_{\omega, q, l ; S}, \quad f \in H_{\omega, q, l ; S} .
$$

Fix $q \in(0,1)$ and $l \in(0, a)$. Let $f$ be an arbitrary function in $H_{\omega, q, l ; S}$. Then

$$
|f(\zeta)| \leq\|f\|_{\omega, q, l ; S} \cdot e^{q \omega(\operatorname{Re} \zeta)+l|\operatorname{Im} \zeta|}, \quad \zeta \in S .
$$

Take $\varepsilon>0$ with $q+2 \varepsilon<1$ and $l+2 \varepsilon<a$. Next, find $k_{0}=k_{0}(q, l)$ such that

$$
\begin{aligned}
2 l \delta_{k} & \leq \varepsilon, \\
2 q \delta_{k}+l\left(1+2 \delta_{k}\right) & \leq l+\varepsilon, \\
q \omega\left(\left(1+2 \delta_{k}\right) t\right) & \leq(q+\varepsilon) \omega(t)+C_{1}, \quad t \geq 0,
\end{aligned}
$$

for all $k \geq k_{0}$. The last condition can be ensured in view of (2.1).

Let $z$ be an arbitrary point of $S_{0}$, i.e., an arbitrary point of the real or the imaginary axis. If $z \in\left[-r_{1}, r_{1}\right] \cup\left[-i r_{1}, i r_{1}\right] \cup\left(i \mathbb{R} \backslash \bigcup_{j} E_{j}\right)$, then $z \in S$, so that $|f(z)|$ satisfies estimate (3.10) with $\zeta=z$.

Consider the remaining cases.

1) Let $z=\operatorname{Re} z,|z|>r_{1}$. We find $k \in \mathbb{N}$ such that $r_{k} \leq|\operatorname{Re} z|<r_{k+1}$. On the corresponding circle $C_{z}^{\mathrm{Re}}$, we choose a point $\zeta$ such that $|f(\zeta)|=\sup \{|f(w)|: w$ is inside $\left.C_{z}^{\mathrm{Re}}\right\}$. Then (3.10), (3.6), and (3.7) show that

$$
|f(z)| \leq|f(\zeta)| \leq\|f\|_{\omega, q, l ; S} \cdot \exp \left(q \omega\left(\left(1+2 \delta_{k}\right) \operatorname{Re} z\right)+2 l \delta_{k} \omega(\operatorname{Re} z)\right) .
$$

For $k \geq k_{0}$, by (3.11) and (3.13) this estimate can be continued as follows:

$$
|f(z)| \leq\|f\|_{\omega, q, l ; S} \cdot e^{C_{1}} \cdot e^{(q+2 \varepsilon) \omega(\operatorname{Re} z)} .
$$


Putting $C_{2}:=\exp \left(C_{1}+q \omega\left(\left(1+2 \delta_{1}\right) r_{k_{0}}\right)+2 l \delta_{1} \omega\left(r_{k_{0}}\right)\right)$, we obtain

$$
|f(z)| \leq C_{2} \cdot\|f\|_{\omega, q, l ; S} \cdot e^{(q+2 \varepsilon) \omega(\operatorname{Re} z)}, \quad z=\operatorname{Re} z,|z|>r_{1} .
$$

2) If $z=i \operatorname{Im} z,|z|>r_{1}$, and $z \in \bigcup_{j} E_{j}$, then, as above, we find $j(z) \in \mathbb{N}$ and $k \in \mathbb{N}$ with $z \in E_{j(z)}$ and $r_{k} \leq|z|<r_{k+1}$. On the boundary of the disk $E_{j(z)}$, we take a point $\zeta$ with $|f(\zeta)|=\sup \left\{|f(w)|: w \in E_{j(z)}\right\}$. Then

$$
|\operatorname{Re} \zeta| \leq 2 \rho_{j(z)}, \quad|\operatorname{Im} \zeta| \leq|\operatorname{Im} z|+2 \rho_{j(z)} .
$$

By (3.10) and (3.9), we have

$$
\begin{aligned}
|f(z)| & \leq|f(\zeta)| \leq\|f\|_{\omega, q, l ; S} \cdot \exp \left\{q \omega\left(2 \rho_{j(z)}\right)+l\left(|\operatorname{Im} z|+2 \rho_{j(z)}\right)\right\} \\
& \leq\|f\|_{\omega, q, l ; S} \cdot \exp \left\{q \omega\left(2 \delta_{k} \operatorname{Im} z\right)+l\left(1+2 \delta_{k}\right)|\operatorname{Im} z|\right\} \\
& \leq\|f\|_{\omega, q, l ; S} \cdot \exp \left\{\left(2 q \delta_{k}+l\left(1+2 \delta_{k}\right)\right)|\operatorname{Im} z|\right\} .
\end{aligned}
$$

Since $k \geq k_{0}$, by (3.12) we obtain

$$
|f(z)| \leq\|f\|_{\omega, q, l ; S} \cdot e^{(l+\varepsilon)|\operatorname{Im} z|} .
$$

Putting $\rho_{0}:=\sup \left\{\rho_{j(z)}:|z|<r_{k_{0}}\right\}$ and $C_{3}:=\exp \left\{q \omega\left(2 \rho_{0}\right)+l\left(r_{k_{0}}+2 \rho_{0}\right)\right\}$, we finally conclude that

$$
|f(z)| \leq C_{3} \cdot\|f\|_{\omega, q, l ; S} \cdot e^{(l+\varepsilon)|\operatorname{Im} z|}, \quad z=i \operatorname{Im} z, \quad|z| \geq r_{1}, \quad z \in \bigcup_{j} E_{j} .
$$

Combining (3.14) and (3.15), we see that

$$
|f(z)| \leq C \cdot\|f\|_{\omega, q, l ; S} \cdot \exp \{(q+2 \varepsilon) \omega(\operatorname{Re} z)+(l+\varepsilon)|\operatorname{Im} z|\}
$$

for $z \in S_{0} \backslash S$, where $C=\max \left\{C_{2}, C_{3}\right\}$ is independent of $f$.

Put $\widetilde{q}=q+2 \varepsilon$ and $\widetilde{l}=l+\varepsilon$. Then $\|f\|_{\omega, \widetilde{q}, \tilde{l} ; S_{0}} \leq C \cdot\|f\|_{\omega, q, l ; S}$. This proves the lemma.

By Epifanov's result (see [12, Theorem 1]) concerning discretization of weakly sufficient sets, the set $S$ contains a subset $\left(\nu_{j}\right)_{j=1}^{\infty},\left|\nu_{j}\right| \uparrow \infty$, weakly sufficient for $H_{(\omega), I}^{1}$. Moreover, from (3.5) and (3.8) it follows that

$$
\left|\mu\left(\nu_{j}\right)\right| \geq e^{-\varepsilon_{k} \omega\left(\operatorname{Re} \nu_{j}\right)-\varepsilon_{k}\left|\operatorname{Im} \nu_{j}\right|}, \quad j \geq j(k),
$$

where $j(k)$ is chosen in such a way that $\left|\nu_{j(k)}\right| \geq r_{k}$.

In conclusion of this section, we apply the result of Korobeinnik about the relationship between weakly sufficient sets and ARS. We recall (see [7]) that a sequence $\left(x_{j}\right)_{j=1}^{\infty}$ of elements of a locally convex space $H$ is called an ARS in $H$ if every element $x \in H$ expands in an absolutely convergent series $x=\sum_{j=1}^{\infty} c_{j} x_{j}$. By [13, Theorem $\mathrm{K}$ ], since the set $\left(\nu_{j}\right)_{j=1}^{\infty}$ is weakly sufficient for $H_{(\omega), I}^{1}$, the system of exponentials $\left\{e^{-i \nu_{j} x}\right\}_{j=1}^{\infty}$ is an $\operatorname{ARS}$ in $\mathcal{E}_{(\omega)}^{1}(I)$.

\section{§4. TheOrem on a PARTiCUlar solution of A CONVOLUtion EQUATION}

Theorem 1. Let $\mu$ be a divisor for $H_{(\omega), I}^{1}$, and let $\left\{e^{-i \nu_{j} x}\right\}_{j=1}^{\infty}$ be the corresponding ARS in $\mathcal{E}_{(\omega)}^{1}(I)$. If the right-hand side $g$ of the convolution equation (1.1) is expanded in a series $g=\sum_{j=1}^{\infty} g_{j} e^{-i \nu_{j} x}$ convergent absolutely in $\mathcal{E}_{(\omega)}^{1}(I)$, then the function

$$
f=\sum_{j=1}^{\infty} \frac{g_{j}}{\mu\left(\nu_{j}\right)} e^{-i \nu_{j} x}
$$

is a particular solution of the convolution equation (1.1) in $\mathcal{E}_{(\omega)}^{1}(I)$ (the last series converges absolutely in $\left.\mathcal{E}_{(\omega)}^{1}(I)\right)$. 
Proof. We show that the series (4.1) converges absolutely in $\mathcal{E}_{(\omega)}^{1}(I)$. It is known (see 9 , Lemma 3]), that

$$
\frac{1}{1+\left|\nu_{j}\right|} \cdot e^{q \omega\left(\nu_{j}\right)+l\left|\operatorname{Im} \nu_{j}\right|} \leq\left|e^{-i \nu_{j} x}\right|_{\omega, q, l} \leq e^{q \omega\left(\nu_{j}\right)+l\left|\operatorname{Im} \nu_{j}\right|}, \quad j \in \mathbb{N},
$$

for all $q \in(0,1)$ and $l \in(0, a)$.

We fix $q \in(0,1)$ and $l \in(0, a)$, and denote $a_{j}:=\frac{\left|g_{j}\right|}{\left|\mu\left(\nu_{j}\right)\right|} \cdot\left|e^{-i \nu_{j} x}\right|_{\omega, q, l}$. Take $\varepsilon>0$ with $q+\varepsilon<1, l+\varepsilon<a$. Then find an index $k_{0}$ such that $\varepsilon_{k}<\varepsilon$ for $k \geq k_{0}$ and choose $j_{0}=j\left(k_{0}\right)$ such that (3.16) is fulfilled for $j \geq j_{0}$.

We estimate $a_{j}$ for $j \geq j_{0}$. Let $k \geq k_{0}$ be such that $r_{k} \leq\left|\nu_{j}\right|<r_{k+1}$. By (3.16) and (4.2), we have

$$
a_{j} \leq\left|g_{j}\right| e^{(q+\varepsilon) \omega\left(\nu_{j}\right)+(l+\varepsilon)\left|\operatorname{Im} \nu_{j}\right|} \leq\left|g_{j}\right| \cdot\left|e^{-i \nu_{j} x}\right|_{\omega, q+2 \varepsilon, l+2 \varepsilon} \cdot e^{\ln \left(1+\left|\nu_{j}\right|\right)-\varepsilon \omega\left(\nu_{j}\right)-\varepsilon\left|\operatorname{Im} \nu_{j}\right|} .
$$

By the restriction $(\gamma)$ on the weight $\omega$, there exists $C>1$ with

$$
\ln (1+t) \leq \varepsilon \omega(t)+\ln C, \quad t \geq 0 .
$$

Therefore, $a_{j} \leq C \cdot\left|g_{j}\right| \cdot\left|e^{-i \nu_{j} x}\right|_{\omega, q+2 \varepsilon, l+2 \varepsilon}, j \geq j_{0}$. Since

$$
\sum_{j=1}^{\infty}\left|g_{j}\right| \cdot\left|e^{-i \nu_{j} x}\right|_{\omega, q+2 \varepsilon, l+2 \varepsilon}<\infty
$$

we see that $\sum_{j=1}^{\infty} a_{j}<\infty$, that is, the series (4.1) converges absolutely in $\mathcal{E}_{(\omega)}^{1}(I)$. Since the space $\mathcal{E}_{(\omega)}^{1}(I)$ is complete, the sum $f$ of this series is a function in $\mathcal{E}_{(\omega)}^{1}(I)$; clearly, it is a solution of the convolution equation (1.1).

\section{§5. A particular and the General solution of the CONVOlution EQUation in THE CASE WHERE $\omega(t)=t^{\rho(t)}$}

Consider the convolution equation (1.1) in the space $\mathcal{E}_{(\omega)}^{1}(I)$ with $\omega(t)=t^{\rho(t)}$, where $\rho(t) \rightarrow \rho \in(0,1)$ is some proximate order. We put $\rho(z):=\rho(|z|), z \in \mathbb{C}$.

Every such weight is strict (see $\S 2$ ), so that all multipliers of the corresponding space $H_{(\omega), I}^{1}$ are strong and all convolution equations (1.1) are differential equations (1.2) with constant coefficients.

Suppose that the symbol $\mu$ of equation (1.2) is determined by its simple zeros:

$$
\mu(z)=\prod_{s=1}^{\infty}\left(1-\frac{z}{\lambda_{s}}\right), \quad\left|\lambda_{s}\right| \uparrow \infty,
$$

and, moreover, the sequence of zeros satisfies the condition

$$
\lim _{s \rightarrow \infty} \frac{s}{\left|\lambda_{s}\right|^{\rho\left(\lambda_{s}\right)}}=0
$$

and forms an R-set (see [14). The last claim means that the points $\lambda_{s}$ lie inside certain angles with common vertex at zero that have no other points in common, and moreover, the points inside one particular angle, when enumerated in the order of increase of their moduli, satisfy

$$
\left|\lambda_{s+1}\right|-\left|\lambda_{s}\right|>d\left|\lambda_{s}\right|^{1-\rho\left(\lambda_{s}\right)}, \quad s \in \mathbb{N}, \text { for some } d>0 .
$$

Formula (5.1) is equivalent to the property that $\mu$ has zero type at the order $\rho(r)$, i.e., is a multiplier for $H_{(\omega), I}^{1}$. In its turn, condition (5.2) ensures that $\mu$ is a divisor of $H_{(\omega), I}^{1}$, i.e., it satisfies (SC). Indeed, if (5.2) is fulfilled, then $\mu$ is of completely regular growth at 
the order $\rho(r)$, that is, outside some exceptional disks $C_{s}=\left\{z:\left|z-\lambda_{s}\right|<r_{s}\right\}, s \in \mathbb{N}$, we have

$$
\lim _{z \rightarrow \infty} \frac{\ln |\mu(z)|}{|z|^{\rho(z)}}=0
$$

It is well known (see [15, the remark after Theorem 1.2.6]), in the case in question any fixed $\gamma>0$ can be taken for the role of all radii $r_{s}$. If $\gamma$ is sufficiently small, the disks $C_{s}$ are mutually disjoint.

Similar symbols $\mu$ that determine resolvable convolution equations in $\mathcal{E}_{(\omega)}^{1}(I)$ were treated in [16].

Clearly, in the case under study, the general solution of the homogeneous equation $\sum_{k=0}^{\infty} a_{k} f^{(k)}=0$ corresponding to (1.2) in $\mathcal{E}_{(\omega)}^{1}(I)$ has the form

$$
f=\sum_{s=1}^{\infty} \alpha_{s} e^{-i \lambda_{s} x}, \quad \alpha_{s} \in \mathbb{C}, \quad s \in \mathbb{N},
$$

and the series in (5.4) converges absolutely in $\mathcal{E}_{(\omega)}^{1}(I)$. Indeed, since the zeros $\lambda_{s}$ of the symbol $\mu$ lie sufficiently far away from one another, there is no need in gathering the corresponding elementary solutions $e^{-i \lambda_{s} x}$ into groups (see $\S 2$ and [2]).

Our aim in this and the next section is to explicitly construct the exponents $\nu_{j}$ of the system $\left\{e^{-i \nu_{j} x}\right\}_{j=1}^{\infty}$ of Theorem 1 in terms of the zeros $\lambda_{s}, s \in \mathbb{N}$ of $\mu$. It should be noted that the $\nu_{j}$ will be chosen on the positive real semiaxis.

In the present section, we shall describe a way of constructing the system $\left(\nu_{j}\right)_{j=1}^{\infty}$ and formulate the results about a particular solution and the general solution for the convolution equation. All justifications will be presented in the next (final) section because they are bulky.

Choose a sufficiently small $\gamma<\frac{1}{4 a}$. Let $C_{s}=\left\{z:\left|z-\lambda_{s}\right|<\gamma\right\}$ be the corresponding exceptional disks such that (5.3) holds true outside them.

The positive zeros $\frac{\pi n}{a}, n \in \mathbb{N}$, of the function $\sin a z$ will be split in two monotone increasing sequences $\xi_{1}, \xi_{2}, \ldots$ and $\eta_{1}, \eta_{2}, \ldots$ in accordance with the following rule: the points $\pm \eta_{j}$ do not belong to any of $C_{s}$, whereas either $\xi_{j}$ or $-\xi_{j}$ belongs to some $C_{s}$. We shall analyze the most difficult case where there are infinitely many points $\xi_{j}$. Put $K_{j}^{ \pm}=\left\{z:\left|z \mp \xi_{j}\right|<\gamma\right\}, j \in \mathbb{N}$.

Next, we apply [15, Theorem 1.2.3] to extract a subsequence $\left(\zeta_{k}\right)_{k=1}^{\infty}$ from the sequence $\left(\frac{\pi}{2 a}+\frac{\pi n}{a}\right)_{n=1}^{\infty}$ so that all points of this subsequence lie outside $\bigcup_{s} C_{s}$ and satisfy

$$
\begin{aligned}
& \lim _{k \rightarrow \infty} \frac{k}{\zeta_{k}^{\rho\left(\zeta_{k}\right)}}=\frac{1}{\pi} \tan \frac{\pi \rho}{2} ; \\
& \zeta_{k+1}-\zeta_{k}>d_{1} \zeta_{k}^{1-\rho\left(\zeta_{k}\right)}, \quad k \in \mathbb{N}, \text { for some } d_{1}>0 .
\end{aligned}
$$

A fortiori, $\zeta_{k} \notin \bigcup_{j} K_{j}^{ \pm}, k \in \mathbb{N}$.

We define the required sequence $\left(\nu_{j}\right)_{j=1}^{\infty}$ to be the union of $\left(\eta_{k}\right)_{k=1}^{\infty}$ and $\left(\zeta_{k}\right)_{k=1}^{\infty}$.

In $\S 6$ it will be proved that, with this choice of the $\nu_{j}, j \in \mathbb{N}$, the system $\left\{e^{-i \nu_{j} x}\right\}_{j=1}^{\infty}$ will be an ARC in $\mathcal{E}_{(\omega)}^{1}(I)$ and, moreover, the quantities $\left|\mu\left(\nu_{j}\right)\right|$ satisfy (3.16). This will establish the following statement.

Theorem 2. Let $\omega(t)=t^{\rho(t)}$, where $\rho(t) \rightarrow \rho \in(0,1)$ is some proximate order. Let $\mu(z)=\prod_{s=1}^{\infty}\left(1-\frac{z}{\lambda_{s}}\right)=\sum_{k=0}^{\infty} a_{k}(-i)^{k} z^{k}$, where the sequence $\left(\lambda_{s}\right)_{s=1}^{\infty}$ satisfies (5.1) and forms an R-set. Finally, let $\left(\nu_{j}\right)_{j=1}^{\infty}$ be the sequence constructed above by the points $\lambda_{s}$.

Suppose that the right-hand side $g$ of equation (1.2) is represented by the series $g=$ $\sum_{j=1}^{\infty} g_{j} e^{-i \nu_{j} x}$ absolutely convergent in $\mathcal{E}_{(\omega)}^{1}(I)$. Then the function $f=\sum_{j=1}^{\infty} \frac{g_{j}}{\mu\left(\nu_{j}\right)} e^{-i \nu_{j} x}$ 
is a particular solution of this equation in the space $\mathcal{E}_{(\omega)}^{1}(I)$, and its general solution has the form

$$
f=\sum_{s=1}^{\infty} \alpha_{s} e^{-i \lambda_{s} x}+\sum_{j=1}^{\infty} \frac{g_{j}}{\mu\left(\nu_{j}\right)} e^{-i \nu_{j} x}, \quad \alpha_{s} \in \mathbb{C}, \quad s \in \mathbb{N} .
$$

Remark 1 . If the zeros $\lambda_{s}$ of the symbol $\mu$ satisfy the condition

$$
\left|\operatorname{Im} \lambda_{s}\right| \geq \delta_{0}, \quad s \geq s_{0},
$$

we can choose the $\eta_{k}$ as follows: $\eta_{k}=\frac{\pi k}{a}, k \in \mathbb{N}$.

We show an example of the construction of the sequence $\left(\nu_{j}\right)_{j=1}^{\infty}$.

Example 1. Let $\omega(t)=t^{1 / 2}$, and let $\mu(z)=\prod_{s=1}^{\infty}\left(1-\frac{z}{\lambda_{s}}\right)$, where $\left(\lambda_{s}\right)_{s=1}^{\infty}$ satisfies (5.1), (5.2), and (5.7). Then the sequence $\left(\nu_{j}\right)_{j=1}^{\infty}$ can be chosen as the union of the sequences

$$
\eta_{k}=\frac{\pi k}{a}, \quad k=1, \ldots, \text { and } \zeta_{k}=\frac{\pi}{2 a}+\frac{\pi\left[a \pi k^{2}\right]}{a}, \quad k=1, \ldots
$$

Here, as usual, $\left[a \pi k^{2}\right]$ is the integral part of $a \pi k^{2}, k \in \mathbb{N}$. In fact, conditions (5.5) and (5.6) are easily verified for $\left(\zeta_{k}\right)_{k=1}^{\infty}$ in this case.

\section{$\S 6$. Proof OF The Results of $\S 5$}

Our main purpose in the present section is to prove that if the $\nu_{j}, j \in \mathbb{N}$, are chosen as described in $\S 5$, then the system $\left\{e^{-i \nu_{j} x}\right\}_{j=1}^{\infty}$ is an ARS in $\mathcal{E}_{(\omega)}^{1}(I)$, and, moreover, the quantities $\left|\mu\left(\nu_{j}\right)\right|, j \in \mathbb{N}$, satisfy (3.16).

By [17. Theorem 3], the system $\left\{e^{-i \nu_{j} x}\right\}_{j=1}^{\infty}$ is an ARS in $\mathcal{E}_{(\omega)}^{1}(I)$ if and only if there exists an entire function $L(z)$ with the following properties:

(A) for every $\varepsilon>0$ there exists $C>0$ such that

$$
|L(z)| \leq C \cdot \exp \left\{(1+\varepsilon)|z|^{\rho(z)}+(a+\varepsilon)|\operatorname{Im} z|\right\}, \quad z \in \mathbb{C} ;
$$

(B) there is a sequence $R_{n} \uparrow \infty$ such that for every $\varepsilon>0$ there exists an index $n_{0}(\varepsilon)$ with

$$
|L(z)| \geq \exp \left\{(1-\varepsilon)|z|^{\rho(z)}+(a-\varepsilon)|\operatorname{Im} z|\right\}, \quad|z|=R_{n}, \quad n \geq n_{0}(\varepsilon)
$$

( $\Gamma)$ for all $q \in(0,1)$ we have

$$
\sum_{j=1}^{\infty} \frac{1}{\left|L^{\prime}\left(\nu_{j}\right)\right|} \exp q \nu_{j}^{\rho\left(\nu_{j}\right)}<\infty .
$$

An entire function $L(z)$ with the above properties will be constructed in the form $L(z)=L_{1}(z) \cdot L_{2}(z)$, where

$$
L_{1}(z)=a z \prod_{k=1}^{\infty}\left(1-\frac{z^{2}}{\eta_{k}^{2}}\right), \quad L_{2}(z)=\prod_{k=1}^{\infty}\left(1-\frac{z^{2}}{\zeta_{k}^{2}}\right)
$$

(the points $\eta_{k}$ and $\zeta_{k}, k \in \mathbb{N}$, were chosen in $\S 5$ ).

We show that $L(z)$ satisfies $(\mathrm{A}),(\mathrm{B})$, and $(\Gamma)$.

First, we study the function $L_{1}(z)$. By construction, $L_{1}(z)=\frac{\sin a z}{\varphi(z)}$, where $\varphi(z)=$ $\prod_{j=1}^{\infty}\left(1-\frac{z^{2}}{\xi_{j}^{2}}\right)$; here the $\xi_{j}, j \in \mathbb{N}$, are the positive zeros of $\sin a z$ with the property that either $\xi_{j}$ or $-\xi_{j}$ belongs to some exceptional disk $C_{s}$ for the symbol $\mu$. Since the diameter of $C_{s}$ is $2 \gamma<\frac{1}{a}$, every $C_{s}$ may contain at most one $\xi_{j}$. Clearly, this implies that, like $\left(\lambda_{s}\right)_{s=1}^{\infty}$, the sequence $\left(\xi_{j}\right)_{j=1}^{\infty}$ forms an R-set and $\lim _{j \rightarrow \infty} \frac{j}{\xi_{j}^{\rho\left(\xi_{j}\right)}}=0$. Therefore, $\varphi$ has 
zero type at the order $\rho(r)$ and is of completely regular growth at this order. Accordingly, outside the disks $K_{j}^{ \pm}=\left\{z:\left|z \mp \xi_{j}\right|<\gamma\right\}$ we have

$$
\lim _{z \rightarrow \infty} \frac{\ln |\varphi(z)|}{|z|^{\rho(z)}}=0 .
$$

Thus, for every $\varepsilon>0$ there exists $r_{0}(\varepsilon)>0$ such that

$$
\begin{aligned}
& |\varphi(z)| \leq e^{\varepsilon|z|^{\rho(z)}}, \quad|z| \geq r_{0}(\varepsilon) ; \\
& |\varphi(z)| \geq e^{-\varepsilon|z|^{\rho(z)}}, \quad|z| \geq r_{0}(\varepsilon), \quad z \notin \bigcup_{j} K_{j}^{ \pm} .
\end{aligned}
$$

Let an index $k_{0}(\varepsilon)$ be chosen in such a way that $\eta_{k} \geq r_{0}(\varepsilon)$ and $\zeta_{k} \geq r_{0}(\varepsilon)$, for $k \geq k_{0}(\varepsilon)$. By (6.1), we obtain

$$
\left|L_{1}^{\prime}\left(\eta_{k}\right)\right|=\frac{a}{\left|\varphi\left(\eta_{k}\right)\right|} \geq a e^{-\varepsilon \eta_{k}^{\rho\left(\eta_{k}\right)}}, \quad k \geq k_{0}(\varepsilon) .
$$

Next, by (6.1) and the choice of the $\zeta_{k}$, we see that

$$
\left|L_{1}\left(\zeta_{k}\right)\right|=\frac{1}{\left|\varphi\left(\zeta_{k}\right)\right|} \geq e^{-\varepsilon \zeta_{k}^{\rho\left(\zeta_{k}\right)}}, \quad k \geq k_{0}(\varepsilon)
$$

Other properties of the function $L_{1}(z)$ are collected in the following lemmas.

Lemma 4. The function $L_{1}(z)$ obeys the upper estimate

$$
\left|L_{1}(z)\right| \leq e^{a \gamma} \cdot \exp \left\{2 \varepsilon|z|^{\rho(z)}+a|\operatorname{Im} z|\right\}, \quad|z| \geq r_{0}(\varepsilon) .
$$

Proof. Since $|\sin a z| \leq e^{a|\operatorname{Im} z|}$ for all $z \in \mathbb{C}$, by (6.2) we see that

$$
\left|L_{1}(z)\right| \leq \exp \left\{\varepsilon|z|^{\rho(z)}+a|\operatorname{Im} z|\right\}, \quad|z| \geq r_{0}(\varepsilon), \quad z \notin K_{j}^{ \pm} .
$$

Now, let $z$ belong to some of the exceptional disks $K_{j}^{ \pm}$, for instance, to $K_{j}^{+}=\{t$ : $\left.\left|t-\xi_{j}\right|<\gamma\right\}$, and let $|z| \geq r_{0}(\varepsilon)$. On the circle $\left|t-\xi_{j}\right|=\gamma$, we find a point $w$ such that $\left|L_{1}(w)\right|=\max \left\{\left|L_{1}(t)\right|:\left|t-\xi_{j}\right| \leq \gamma\right\}$. At the same time, we have $|w| \leq|z|+2 \gamma$, $|\operatorname{Im} w| \leq|\operatorname{Im} z|+\gamma$. Increasing $r_{0}(\varepsilon)$ if necessary, we may and do assume that

$$
(t+2 \gamma)^{\rho(t+2 \gamma)} \leq 2 t^{\rho(t)}, \quad t \geq r_{0}(\varepsilon)
$$

Consequently,

$$
\left|L_{1}(z)\right| \leq\left|L_{1}(w)\right| \leq \exp \left\{\varepsilon|w|^{\rho(w)}+a|\operatorname{Im} w|\right\} \leq e^{a \gamma} \cdot \exp \left\{2 \varepsilon|z|^{\rho(z)}+a|\operatorname{Im} z|\right\} .
$$

This proves the lemma.

Lemma 5. On the circles $|z|=R_{n}$, where $R_{n}=\frac{\pi}{4 a}+\frac{\pi n}{a}, n \in \mathbb{N}$, we have

$$
\left|L_{1}(z)\right| \geq M \cdot \exp \left\{-\varepsilon|z|^{\rho(z)}+a|\operatorname{Im} z|\right\}, \quad n \geq n_{0}(\varepsilon),
$$

where $M:=\frac{1}{2}\left(1-e^{-\frac{\pi}{4}}\right)$.

Proof. Let $n_{0}(\varepsilon)$ be such that $R_{n} \geq r_{0}(\varepsilon)$ for $n \geq n_{0}(\varepsilon)$. Then $|\varphi(z)|$ obeys (6.1) on the circles $|z|=R_{n}, n \geq n_{0}(\varepsilon)$.

We estimate $\left|\sin \operatorname{are}^{i \theta}\right|$ for $r=R_{n}, n \in \mathbb{N}$. Clearly, it suffices to consider $\theta \in\left[0, \frac{\pi}{2}\right]$. We have

$$
\left|\sin a r e^{i \theta}\right|=\frac{1}{2} A(r, \theta) \cdot e^{a r \sin \theta}, \quad r \geq 0, \quad \theta \in\left[0, \frac{\pi}{2}\right],
$$

where $A(r, \theta)=\left|1-e^{-2 \operatorname{ar}(\sin \theta-i \cos \theta)}\right|$. The quantity $A(r, \theta)$ satisfies the following inequalities:

$$
A(r, \theta) \geq 1-e^{-2 a r \sin \theta} ; \quad A(r, \theta) \geq \sqrt{1-\cos ^{2}(2 a r \cos \theta)} .
$$


Fix $n \in \mathbb{N}$. If $\theta \in\left[0, \frac{\pi}{2}\right]$ is such that $2 a R_{n} \sin \theta \leq \frac{\pi}{4}$, then

$$
\frac{\pi}{4}+2 \pi n=2 a R_{n}-\frac{\pi}{4} \leq 2 a R_{n} \cos \theta \leq 2 a R_{n}=\frac{\pi}{2}+2 \pi n,
$$

so that $A\left(R_{n}, \theta\right) \geq \sqrt{1-\frac{1}{2}}=\frac{1}{\sqrt{2}}$ for $\operatorname{such} \theta$. But if $\theta \in\left[0, \frac{\pi}{2}\right]$ is such that $2 a R_{n} \sin \theta>\frac{\pi}{4}$, then $A\left(R_{n}, \theta\right) \geq 1-e^{-\frac{\pi}{4}}$. In any case, $A\left(R_{n}, \theta\right) \geq 2 M, \theta \in\left[0, \frac{\pi}{2}\right]$. Thus,

$$
|\sin a z| \geq M e^{a|\operatorname{Im} z|}, \quad|z|=R_{n}, \quad n \in \mathbb{N} .
$$

Combining this with (6.1), we arrive at (6.6).

We pass to the study of $L_{2}(z)$. By (5.6),$L_{2}(z)$ is a function of completely regular growth at the order $\rho(r)$. Next, taking (5.5) and [15, Theorem 1.2.7] into account, we conclude that the indicator of $L_{2}(z)$ at this order is equal to

$$
H_{2}(\theta)=\frac{\cos \rho\left(\theta-\frac{\pi}{2}\right)}{\cos \frac{\rho \pi}{2}}, \quad \theta \in(0, \pi) .
$$

So, increasing $r_{0}(\varepsilon)$ if necessary, we may assume that

$$
\begin{aligned}
\mid L_{2}\left(r e^{i \theta} \mid\right. & \leq \exp \left\{\left(\frac{\cos \rho\left(\theta-\frac{\pi}{2}\right)}{\cos \frac{\rho \pi}{2}}+\varepsilon\right) r^{\rho(r)}\right\}, \quad r \geq r_{0}(\varepsilon), \quad \theta \in[0, \pi] ; \\
\mid L_{2}\left(r e^{i \theta} \mid\right. & \geq \exp \left\{\left(\frac{\cos \rho\left(\theta-\frac{\pi}{2}\right)}{\cos \frac{\rho \pi}{2}}-\varepsilon\right) r^{\rho(r)}\right\}, \\
r & \geq r_{0}(\varepsilon), \quad \theta \in[0, \pi], \quad r e^{i \theta} \notin \bigcup_{l} B_{l}^{ \pm},
\end{aligned}
$$

where the $B_{l}^{ \pm}=\left\{z:\left|z \mp \zeta_{l}\right|<\gamma\right\}, l \in \mathbb{N}$, are the exceptional disks for $L_{2}(z)$. In particular, since no $\eta_{k}$ lies in $B_{l}^{ \pm}, k, l \in \mathbb{N}$, we obtain

$$
\left|L_{2}\left(\eta_{k}\right)\right| \geq e^{(1-\varepsilon) \eta_{k}^{\rho\left(\eta_{k}\right)}}, \quad k \geq k_{0}(\varepsilon)
$$

Next, by [15, Theorem 1.2.8] we have

$$
\lim _{k \rightarrow \infty} \frac{\ln \frac{1}{\left|L_{2}^{\prime}\left(\zeta_{k}\right)\right|}}{\zeta_{k}^{\rho\left(\zeta_{k}\right)}}=-1 .
$$

Consequently, $k_{0}(\varepsilon)$ can be chosen so large that

$$
\left|L_{2}^{\prime}\left(\zeta_{k}\right)\right|>e^{(1-\varepsilon) \zeta_{k}^{\rho\left(\zeta_{k}\right)}}, \quad k \geq k_{0}(\varepsilon)
$$

Finally, we prove that the function $L(z)=L_{1}(z) \cdot L_{2}(z)$ possesses the required properties.

Lemma 6. The function $L(z)=L_{1}(z) \cdot L_{2}(z)$ has properties $(\mathrm{A}),(\mathrm{B})$, and $(\Gamma)$.

Proof. (A) Combining (6.5) and (6.7), we see that

$$
\mid L\left(r e^{i \theta} \mid \leq e^{a \gamma} \cdot \exp \left\{\left(\frac{\cos \rho\left(\theta-\frac{\pi}{2}\right)}{\cos \frac{\rho \pi}{2}}+3 \varepsilon\right) r^{\rho(r)}+a r \sin \theta\right\}\right.
$$

for all $r \geq r_{0}(\varepsilon)$ and $\theta \in[0, \pi]$. We find $\theta_{0}(\varepsilon) \in\left(0, \frac{\pi}{2}\right)$ with

$$
1-\varepsilon<\frac{\cos \rho\left(\theta-\frac{\pi}{2}\right)}{\cos \frac{\rho \pi}{2}}<1+\varepsilon, \theta \in\left[0, \theta_{0}(\varepsilon)\right] \cup\left[\pi-\theta_{0}(\varepsilon), \pi\right] .
$$

Then for $\theta$ as in this formula, we have

$$
\mid L\left(r e^{i \theta} \mid \leq e^{a \gamma} \cdot \exp \left\{(1+4 \varepsilon) r^{\rho(r)}+a r \sin \theta\right\}, \quad r \geq r_{0}(\varepsilon) .\right.
$$


But if $\theta \in\left[\theta_{0}(\varepsilon), \pi-\theta_{0}(\varepsilon)\right]$, then, again increasing $r_{0}(\varepsilon)$ if necessary, we obtain

$$
\left(\frac{\cos \rho\left(\theta-\frac{\pi}{2}\right)}{\cos \frac{\rho \pi}{2}}+3 \varepsilon\right) r^{\rho(r)} \leq\left(\frac{1}{\cos \frac{\rho \pi}{2}}+3 \varepsilon\right) r^{\rho(r)} \leq \varepsilon r \sin \theta_{0}(\varepsilon) \leq \varepsilon r \sin \theta
$$

whenever $r \geq r_{0}(\varepsilon)$. Thus, in this case

$$
\left|L\left(r e^{i \theta}\right)\right| \leq e^{a \gamma} \cdot e^{(a+\varepsilon) r \sin \theta}, \quad r \geq r_{0}(\varepsilon) .
$$

This proves $(\mathrm{A})$.

(B) Consider the circles $|z|=R_{n}, R_{n}=\frac{\pi}{4 a}+\frac{\pi n}{a}, n \in \mathbb{N}$. Clearly, they do not intersect the exceptional disks $B_{l}^{ \pm}, l \in \mathbb{N}$. Consequently, by (6.6) and (6.8), for $n \geq n_{0}(\varepsilon)$ on this circles we have the inequalities

$$
\left|L\left(R_{n} e^{i \theta}\right)\right| \geq M \exp \left\{\left(\frac{\cos \rho\left(\theta-\frac{\pi}{2}\right)}{\cos \frac{\rho \pi}{2}}-2 \varepsilon\right) R_{n}^{\rho\left(R_{n}\right)}+a R_{n} \sin \theta\right\}, \quad \theta \in[0, \pi] .
$$

Using the left inequality in (6.11), we see that

$$
\left|L\left(R_{n} e^{i \theta}\right)\right| \geq M \exp \left\{(1-3 \varepsilon) R_{n}^{\rho\left(R_{n}\right)}+a R_{n} \sin \theta\right\}
$$

for

$$
\theta \in\left[0, \theta_{0}(\varepsilon)\right] \cup\left[\pi-\theta_{0}(\varepsilon), \pi\right]
$$

and $n \geq n_{0}(\varepsilon)$.

But if $\theta \in\left[\theta_{0}(\varepsilon), \pi-\theta_{0}(\varepsilon)\right]$, then, taking $n_{0}(\varepsilon)$ so large that

$$
(1+2 \varepsilon) R_{n}^{\rho\left(R_{n}\right)}<\varepsilon R_{n} \sin \theta_{0}(\varepsilon), \quad n \geq n_{0}(\varepsilon),
$$

we get

$$
\begin{aligned}
& \left(\frac{\cos \rho\left(\theta-\frac{\pi}{2}\right)}{\cos \frac{\rho \pi}{2}}-2 \varepsilon\right) R_{n}^{\rho\left(R_{n}\right)}+a R_{n} \sin \theta \geq-2 \varepsilon R_{n}^{\rho\left(R_{n}\right)}+a R_{n} \sin \theta \\
& \quad \geq R_{n}^{\rho\left(R_{n}\right)}+(a-\varepsilon) R_{n} \sin \theta-(1+2 \varepsilon) R_{n}^{\rho\left(R_{n}\right)}+\varepsilon R_{n} \sin \theta_{0}(\varepsilon) \\
& \quad \geq R_{n}^{\rho\left(R_{n}\right)}+(a-\varepsilon) R_{n} \sin \theta
\end{aligned}
$$

Combining the two cases considered above and using again the identity $L(-z)=L(z)$, $z \in \mathbb{C}$, finally we obtain the estimate

$$
|L(z)| \geq M \exp \left\{(1-3 \varepsilon)|z|^{\rho(z)}+(a-\varepsilon)|\operatorname{Im} z|\right\}
$$

on the circles $|z|=R_{n}, n \geq n_{0}(\varepsilon)$. Thus, $L(z)$ obeys condition (B).

( $\Gamma)$ Finally, we estimate $\left|L^{\prime}\left(\eta_{k}\right)\right|$ and $\left|L^{\prime}\left(\zeta_{k}\right)\right|, k \in \mathbb{N}$. We have

$$
L^{\prime}\left(\eta_{k}\right)=L_{1}^{\prime}\left(\eta_{k}\right) \cdot L_{2}\left(\eta_{k}\right), \quad L^{\prime}\left(\zeta_{k}\right)=L_{1}\left(\zeta_{k}\right) \cdot L_{2}^{\prime}\left(\zeta_{k}\right), \quad k \in \mathbb{N} .
$$

By (6.3) and (6.9), we obtain

$$
\left|L^{\prime}\left(\eta_{k}\right)\right| \geq a \cdot e^{(1-2 \varepsilon) \eta_{k}^{\rho\left(\eta_{k}\right)}}, \quad k \geq k_{0}(\varepsilon) .
$$

Similarly, (6.4) and (6.10) yield

$$
\left|L^{\prime}\left(\zeta_{k}\right)\right| \geq e^{(1-2 \varepsilon) \zeta_{k}^{\rho\left(\zeta_{k}\right)}}, \quad k \geq k_{0}(\varepsilon) .
$$

Clearly, these inequalities ensure $(\Gamma)$, and the lemma follows.

The author is grateful to the referee for high attention to this work. 


\section{References}

[1] D. A. Abanina, Solvability of convolution equations in Beurling spaces of ultradifferentiable functions of normal type on an interval, Sibirsk. Mat. Zh. 53 (2012), no. 3, 477-494; English transl., Sib. Math. J. 53 (2012), no. 3, 377-392. MR.2978455

[2] _ Exponential-polynomial basis in the null space of convolution operators in classes of ultradifferentiable functions, Vladikavkaz. Mat. Zh. 13 (2011), no. 4, 3-17. (Russian) MR2907216 (2012m:44005)

[3] R. Meise, B. A. Taylor, and D. Vogt, Equivalence of slowly decreasing conditions and local Fourier expansions, Indiana Univ. Math. J. 36 (1987), no. 4, 729-756. MR.916742 (89c:46058)

[4] R. Meise, K. Schwerdtfeger, and B. A. Taylor, Kernels of slowly decreasing convolution operators, Doğa Mat. 10 (1986), no. 1, 176-197. MR872875 (88e:30073)

[5] J. Bonet, A. Galbis, and R. Meise, On the range of convolution operators on non-quasianalytic ultradifferentiable functions, Studia Math. 126 (1997), no. 2, 171-198. MR.1472697(99a:46071)

[6] A. F. Leont'ev, Representation of arbitrary entire functions by Dirichlet series, Dokl. Akad. Nauk SSSR 164 (1965), no. 1, 40-42. (Russian) MR0188440 (32:5878)

[7] Yu. F. Korobernik, Representative systems, Izv. Akad. Nauk. SSSR Ser. Mat. 42 (1978), no. 2, 325-355; English transl., Math. USSR-Izv. 12 (1978), no. 2, 309-355. MR494558(82b:46006)

[8] D. A. Abanina, Representation of solutions of convolution equations in nonquasianalytic Beurling classes of ultradifferentiable functions of normal type, Izv. Vyssh. Uchebn. Zaved. Mat. 2011, no. 6, 3-11; English transl., Russian Math. (Iz. VUZ) 55 (2011), no. 6, 1-8. MR2931699

[9] A. V. Abanin and I. A. Fulip'ev, Analytic realization of spaces dual to spaces of infinitely differentiable functions, Sibirsk. Mat. Zh. 47 (2006), no. 3, 485-500; English transl., Sib. Math. J. 47 (2006), no. 3, 397-409. MR2249161 (2007h:46035)

[10] D. M. Schneider, Sufficient sets for some spaces of entire functions, Trans. Amer. Math. Soc. 197 (1974), 161-180. MR0357835 (50:10301)

[11] R. P. Boas, Entire functions, Acad. Press, New York, 1954. MR0068627 (16:914f)

[12] O. V. Epifanov, Variations of weakly sufficient sets in spaces of analytic functions, Izv. Vyssh. Uhebn. Zaved. Mat. 1986, no. 7, 50-56; English transl., Sov. Math. (Iz. VUZ) 30 (1986), no. 7, 67-74. MR867608 (88b:30079)

[13] Yu. F. Korobeı̆nik, Inductive and projective topologies. Sufficient sets and representative systems, Izv. Akad. Nauk SSSR Ser. Mat. 50 (1986), no. 3, 539-565; English transl., Math. USSR-Izv. 28 (1987), no. 3, 529-554. MR854595 (88b:46044b)

[14] B. Ya. Levin, Distribution of zeros of entire functions, Gosudarstv. Izdat. Tehn.-Teor. Lit., Moscow, 1956; English transl., Transl. Math. Monogr., vol. 5, Amer. Math. Soc., Providence, RI, 1972. MR0087740 (19:402c)

[15] A. F. Leont'ev, Exponential series, Nauka, Moscow, 1976. (Russian) MR0584943 (58:28451)

[16] D. A. Polyakova, On the continuous linear right inverse for convolution operators in spaces of ultradifferentiable functions, Mat. Zametki 96 (2014), no. 4, 548-566; English transl., Math. Notes 96 (2014), no. 4, 522-537).

[17] A. V. Abanin, Nontrivial expansions of zero and absolutely representing systems, Mat. Zametki 57 (1995), no. 3-4, 335-344. MR1346649 (96e:46031)

Southern Federal University, ul. Mil'Chakova 8A, Rostov-on-Don 344090, Russia

Southern Mathematical Institute, VNTS RAS and RNO-A, ul. Markusa 22, Vladikavkaz 362027, Russia

E-mail address: forsites1@mail.ru

Received 19/MAY/2014

Translated by S. V. KISLYAKOV 